\title{
Photonic modes in synthetic photonic lattices localized due to nontrivial gauge field circulation
}

\section{Artem Pankov, Ilya Vatnik, Dmitry Churkin, Andrey A. Sukhorukov}

Artem Pankov, llya Vatnik, Dmitry Churkin, Andrey A. Sukhorukov, "Photonic modes in synthetic photonic lattices localized due to nontrivial gauge field circulation," Proc. SPIE 10457, AOPC 2017: Laser Components, Systems, and Applications, 1045734 (24 October 2017); doi: 10.1117/12.2285492

SPIE. Event: Applied Optics and Photonics China (AOPC2017), 2017, Beijing, China 


\title{
Photonic Modes in Synthetic Photonic Lattices Localized due to Nontrivial Gauge Field Circulation
}

\author{
Artem Pankov *a , Ilya Vatnik ${ }^{\mathrm{a}}$, Dmitry Churkin ${ }^{\mathrm{a}}$, Andrey A. Sukhorukov ${ }^{\mathrm{b}}$ \\ ${ }^{a}$ Novosibirsk State University, Pirogova St. 2, Novosibirsk, Russia, 630090; \\ ${ }^{\mathrm{b}}$ Nonlinear Physics Centre, Research School of Physics and Engineering, The Australian National \\ University, Canberra ACT 0200, Australia
}

\begin{abstract}
One of concepts giving opportunities for studying of topological insulators in non-magnetic materials, or creating scattering-immune in optical waveguides is creation of synthetic gauge fields in photonic systems. It was shown that gauge fields shift the band-gaps of optical waves, which can be applied to implement one-way nonreciprocal waveguides, even though both the waveguide core and cladding are in a topologically trivial state [1]. In our work we propose a method to create a gauge field in a synthetic photonic mesh lattice - an optical device proved its high versatility for optical experiments [2]. We demonstrate presence of localized modes due to nontrivial gauge field circulation.
\end{abstract}

Keywords: Localized modes, Surface waves, Synthetic Photonic Lattice

\section{INTRODUCTION}

Photonic lattices are a versatile platform for realizing and observing numerous optical phenomena. This platform can provide such properties and possibilities, which no other system can offer. One of representatives of this class is a synthetic photonic lattice (SPL) [2], which is the optical-fiber based system, comprising two fiber loops that are coupled via a directional coupler with the coupling ratio $\theta$. These two fiber loops differ in length by $\Delta \mathrm{L}$, thus a single pulse launched into the system produce a train of pulses circulating, interfering and multiplying within the system. The synthetic photonic lattice is a full analogue of a photonic mesh lattice but realized in time domain rather than in space domain (fig.1). Unlike ordinary waveguide arrays, light propagation such systems is discretized in two dimensions (transverse and longitudinal). The band structure of this family of lattices is derived analytically and its effects on light dynamics are investigated [3]. Phase modulation plays role of an optical potential in the SPL. Apart from the conventional mesh photonic lattices, this optical potential can be easily and precisely controlled by means of an electrooptical modulator together with electrical waveform generator. Up to date, a number of different phenomena including Bloch oscillations [3], Anderson localization [4, 5], PT symmetry breaking [6] were realized in this system.

Creation of synthetic gauge fields for photons in SPL opens new opportunities for realization of robust scatteringimmune waveguides and topological insulators in non-magnetic materials. We predict a new type of localized photonic modes at an interface between two regions with different synthetic gauge fields, even in the absence of band-gap shift.

\section{SPL ANALYTICAL DESCRIPTION}

System of equations governing synthetic photonic lattices can be found from general physical considerations. As it is seen from the figure 2, the system of equations describing pulses evolution in SPL is as follows:

$$
\left\{\begin{array}{l}
u_{n}^{m+1}=e^{i \psi_{n}}\left(\cos (\theta) u_{n+1}^{m}+i \sin (\theta) v_{n+1}^{m}\right) \\
v_{n}^{m+1}=e^{i \varphi_{n}\left(\cos (\theta) v_{n-1}^{m}+i \sin (\theta) u_{n-1}^{m}\right)}
\end{array}\right.
$$

Where $u_{n}^{m}$ - signal intensity at the short loop during round trip $\mathrm{m}$ and at the slot number $\mathrm{n}, v_{n}^{m}$ - same, but at the long loop, $\varphi_{n^{-}}$phase shift at the slot number $\mathrm{n}$ on the short loop, $\psi_{\mathrm{n}}$ - same, but at the long loop and $\theta$ is the coupling coefficient.

AOPC 2017: Laser Components, Systems, and Applications, edited by Shibin Jiang, Lijun Wang,

Lan Jiang, Long Zhang, Proc. of SPIE Vol. 10457, 1045734 - (c) 2017 SPIE

CCC code: $0277-786 \mathrm{X} / 17 / \$ 18 \cdot$ doi: $10.1117 / 12.2285492$ 


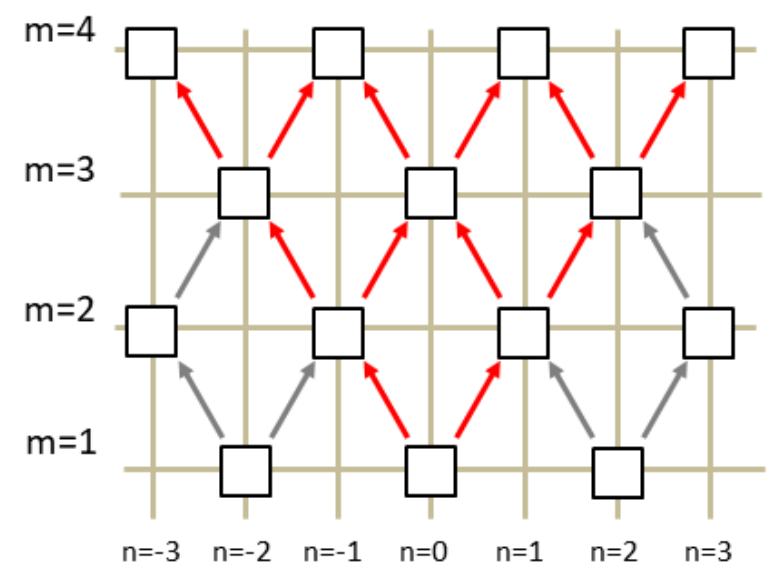

a)

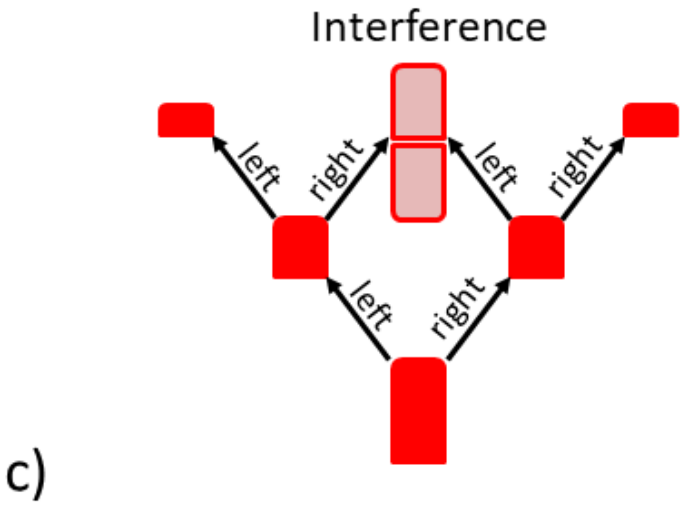

\section{Spatial domain}

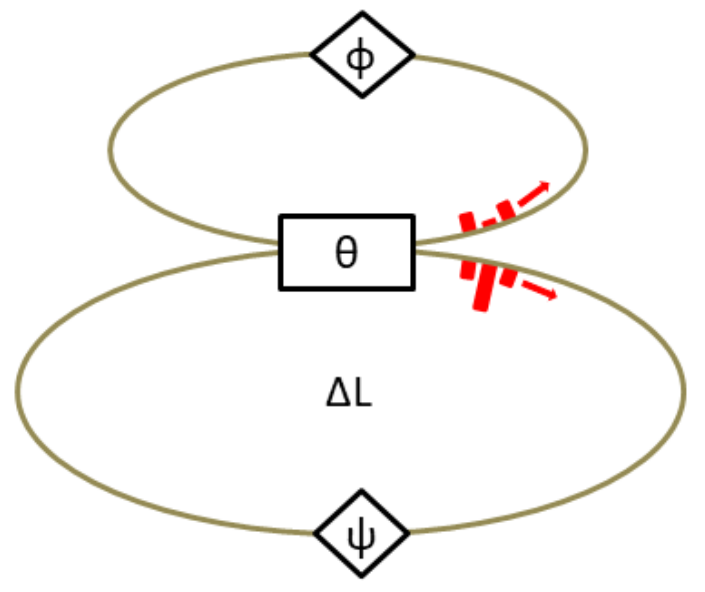

b)

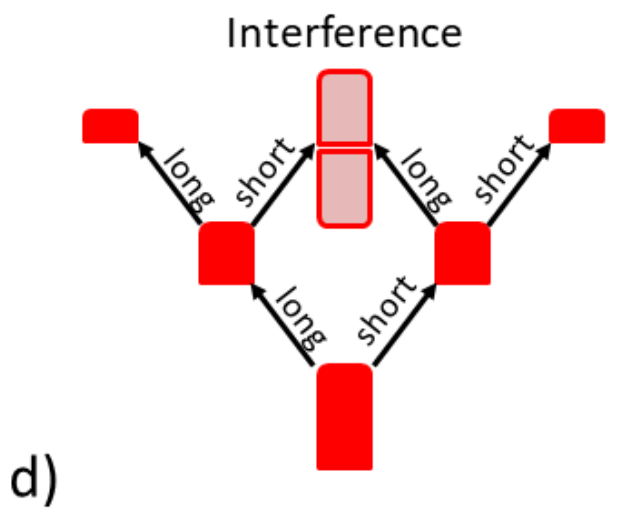

\section{Time domain}

Figure 1. a) Discrete mesh lattice in the spatial domain b) Synthetic photonic lattice which is time-domain equivalent to the discrete mesh lattice c) Pulses propagation in the discrete mesh lattice in the spatial domain d) Pulses evolution in short (long) loop in time.

In case if in synthetic photonic lattice phase is randomly distributed along the coordinate $n$ and invariable along the coordinate $m$, functions $\varphi$ and $\psi$ becomes independent of $m$ and eigenmodes structure of such system can be found. Eigenmodes of such system can be written in the form of functions, similar to Bloch functions: $u_{n}^{m}=U_{n} \exp (i m \beta)$ - for a short loop, $v_{n}^{m}=V_{n} \exp (\mathrm{im} \beta)$ - for a long loop, where $\beta$ is propagation constant.

Then, considering assumption about the eigenmodes form, system of the equations for the two loops system with a random phase shift in each loop and an arbitrary coefficient of the fiber coupler can be written as follows: 


$$
\left(\begin{array}{cc}
U U & U V \\
V U & V V
\end{array}\right) *\left(\begin{array}{c}
u_{1}^{m} \\
u_{2}^{m} \\
\vdots \\
u_{N}^{m} \\
v_{1}^{m} \\
v_{2}^{m} \\
\vdots \\
v_{N}^{m}
\end{array}\right)=\left(\begin{array}{c}
u_{1}^{m+1} \\
u_{2}^{m+1} \\
\vdots \\
u_{N}^{m+1} \\
v_{1}^{m+1} \\
v_{2}^{m+1} \\
\vdots \\
v_{N}^{m+1}
\end{array}\right)=\lambda *\left(\begin{array}{c}
u_{1}^{m} \\
u_{2}^{m} \\
\vdots \\
u_{N}^{m} \\
v_{1}^{m} \\
v_{2}^{m} \\
\vdots \\
v_{N}^{m}
\end{array}\right)
$$

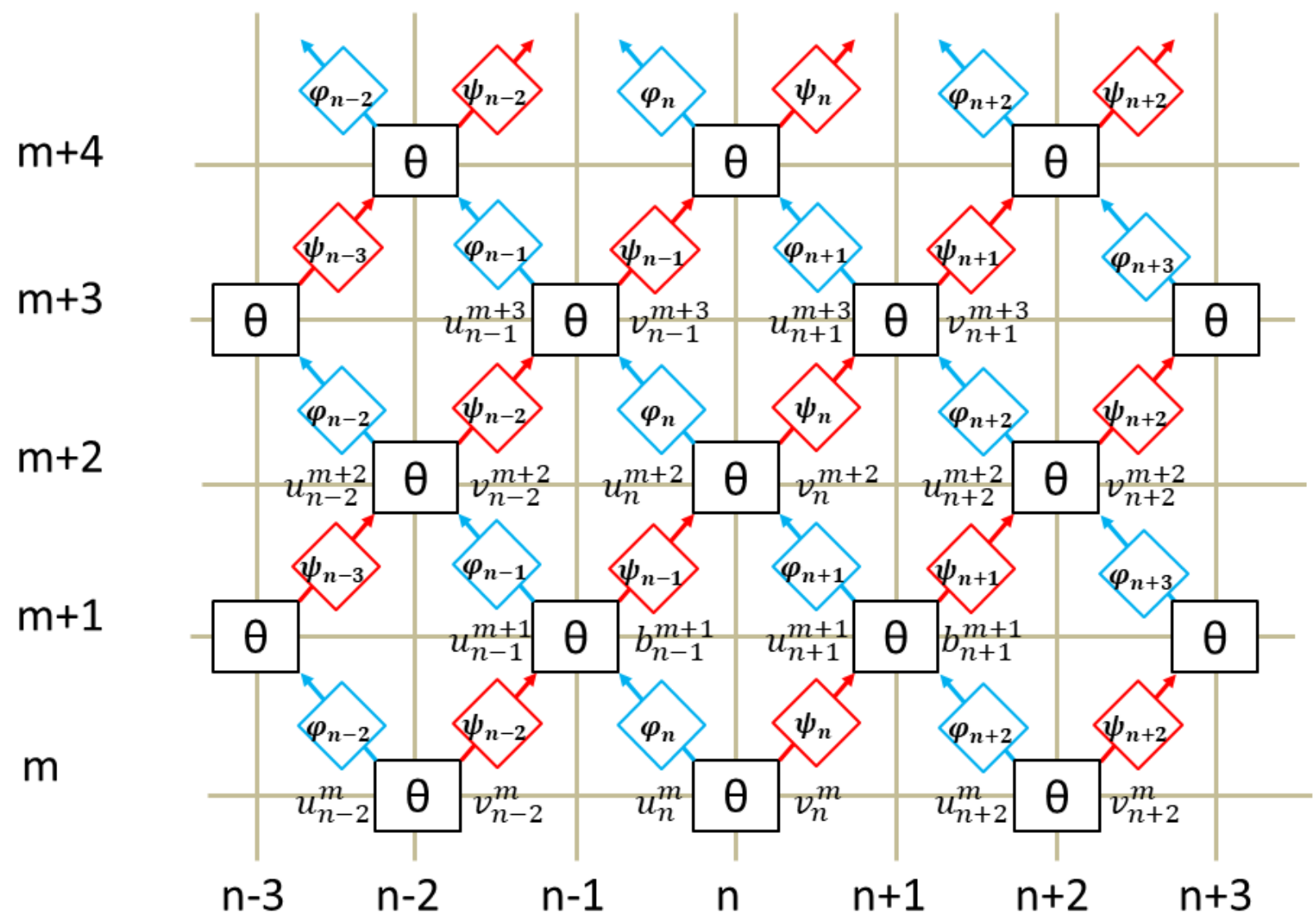

Figure 2. SPL discrete representation. Rhombuses indicate the position of phase elements and squares indicate the coupling regions. Red color indicates propagation in the long loop, and blue -- in the short loop.

, where matrices UU, UV, VU, VV - are defined as follows: 


$$
\begin{aligned}
& U U=\left(\begin{array}{ccccc}
0 & 0 & 0 & \cdots & \cos (\theta) \exp \left(i \psi_{1}\right) \\
\cos (\theta) \exp \left(i \psi_{2}\right) & 0 & 0 & \cdots & 0 \\
\vdots & \cos (\theta) \exp \left(i \psi_{3}\right) & 0 & \ldots & 0 \\
\vdots & \vdots & \ddots & \ddots & \vdots \\
0 & \cdots & 0 & \cos (\theta) \exp \left(i \psi_{N}\right) & 0
\end{array}\right) \\
& U V=\left(\begin{array}{ccccc}
0 & 0 & 0 & \ldots & i \sin (\theta) \exp \left(i \psi_{1}\right) \\
i \sin (\theta) \exp \left(i \psi_{2}\right) & 0 & 0 & \ldots & 0 \\
\vdots & i \sin (\theta) \exp \left(i \psi_{3}\right) & 0 & \ldots & 0 \\
\vdots & \vdots & \ddots & \ddots & \vdots \\
0 & \ldots & 0 & i \sin (\theta) \exp \left(i \psi_{N}\right) & 0
\end{array}\right) \\
& V U=\left(\begin{array}{ccccc}
0 & i \sin (\theta) \exp \left(i \varphi_{1}\right) & 0 & \ldots & 0 \\
0 & 0 & i \sin (\theta) \exp \left(i \varphi_{2}\right) & \ldots & \vdots \\
\vdots & \vdots & 0 & \ddots & 0 \\
0 & \vdots & \vdots & \ddots & i \sin (\theta) \exp \left(i \varphi_{N-1}\right) \\
i \sin (\theta) \exp \left(i \varphi_{N}\right) & 0 & \ldots & 0 & 0
\end{array}\right) \\
& V V=\left(\begin{array}{ccccc}
0 & \cos (\theta) \exp \left(i \varphi_{1}\right) & 0 & \cdots & 0 \\
0 & 0 & \cos (\theta) \exp \left(i \varphi_{2}\right) & \ldots & \vdots \\
\vdots & \vdots & 0 & \ddots & 0 \\
0 & \vdots & \vdots & \ddots & \cos (\theta) \exp \left(i \varphi_{N-1}\right) \\
\cos (\theta) \exp \left(i \varphi_{N}\right) & 0 & \ldots & 0 & 0
\end{array}\right)
\end{aligned}
$$

Solving matrix equation (2) eigenvalues and eigenvectors of this system can be found. Eigenvalue and corresponding eigenvector form an eigenmode, and propagation constants $\beta_{\mathrm{j}}=\log \left(\lambda_{\mathrm{j}}\right) / i$ versus modenumber $\mathrm{j}$ form a dispersion curve. To present system (1) as a matrix equation (2) it was necessary to enter the boundary conditions. They are provided by terms $(1, \mathrm{~N})$ in matrices $\mathrm{UU}$ and $\mathrm{VV}$, and by terms $(\mathrm{N}, 1)$ in matrices $\mathrm{VU}$ and $\mathrm{VV}$.

When phase shifts on both loops are constant $\left(\varphi_{n}^{m}=\varphi, \psi_{n}^{m}=\psi\right)$ then it is possible to derivate an analytical formula for propagation constant $\beta$. In this case, solutions of the matrix equation (2) can be formulated as:

$$
u_{n}^{m}=U e^{i K n+i m \beta}, v_{n}^{m}=V e^{i K n+i m \beta}
$$

where $\mathrm{K}$ is a transverse wavenumber. Substituting eq. (3) into the eq. (2) we obtain an eigenmode equation:

$$
\left(\begin{array}{cc}
e^{i(\varphi-K) \cos (\theta)} & i e^{i(\varphi-K) \sin (\theta)} \\
i e^{i(\psi+K) \sin (\theta)} & e^{i(\psi+K) \cos (\theta)}
\end{array}\right)\left(\begin{array}{l}
U \\
V
\end{array}\right)=e^{i \beta}\left(\begin{array}{l}
U \\
V
\end{array}\right)
$$

Its solution is found as:

$$
\exp \left(\beta_{ \pm}\right)=\exp \left(i\left(\frac{\varphi+\psi}{2}\right)\left[\cos (K+\psi-\varphi) \cos (\theta) \pm \sqrt{\cos ^{2}(K+\psi-\varphi) \cos ^{2}(\theta)-1}\right]\right)
$$

And finally:

$$
\beta_{ \pm}=\frac{\varphi+\psi}{2} \pm \cos ^{-1}[\cos (K+\psi-\varphi) \cos (\theta)]
$$


This formula is responsible for the shape of the dispersion curve.

We note that the effect of phase modulation is twofold. On the one hand, it leads to a shift of propagation constant by $\Delta \beta=(\varphi+\psi) / 2$ similar to modification of a refractive index. On the other hand, $\Delta K=\psi-\varphi$ determines a shift of the wavenumber, which is related to an effective gauge field previously considered for other types of photonic structures [1]. Interestingly, for out-of-phase modulation, $\varphi=-\psi$, there appears effective gauge field yet no band-gap shift, and this new regime could not be realized in previously studied mesh lattices with phase modulation in one loop.

\section{GAUGE FIELD NONTRIVIAL CIRCULATION}

We consider an effective gauge field jump, created by the phase modulation profiles $\varphi_{\mathrm{n}<0}=\psi_{\mathrm{n}<0}=0, \varphi_{\geq 0}=\varphi_{0}, \psi_{\geq 0}=\psi_{0}$, as illustrated on figure 3. We also visualize the effect of phase modulation on a mesh graph [2]. In this scheme, square symbols denote fiber couplers, and arrows represent propagation through a short or long fiber loop, as indicated by colors blue and red respectively. It is established that circulation of the gauge potential can give rise to an effective magnetic field [7]. In order to quantify this phenomenon, we calculate the total phase accumulation $\left(\Xi_{n}\right)$ around closed circles enclosing sets of four nearest beam-splitters. This quantity vanishes for $n \leq-2$ and $n \geq 1$, which is expected as in these regions the phase modulation and the associated gauge fields are constant. However, we find that $\Xi_{-1}=\varphi_{0}$ and $\Xi_{0}=$ $\psi_{0}$, i.e. nontrivial circulation of gauge field appears next to the interface, which is illustrated by circular arrows in figure 3. At these locations, the phase accumulation depends on the order of pulse propagation through the loops, i.e. it is different for short-long or long-short paths, analogous to the Aharonov-Bohm effect.

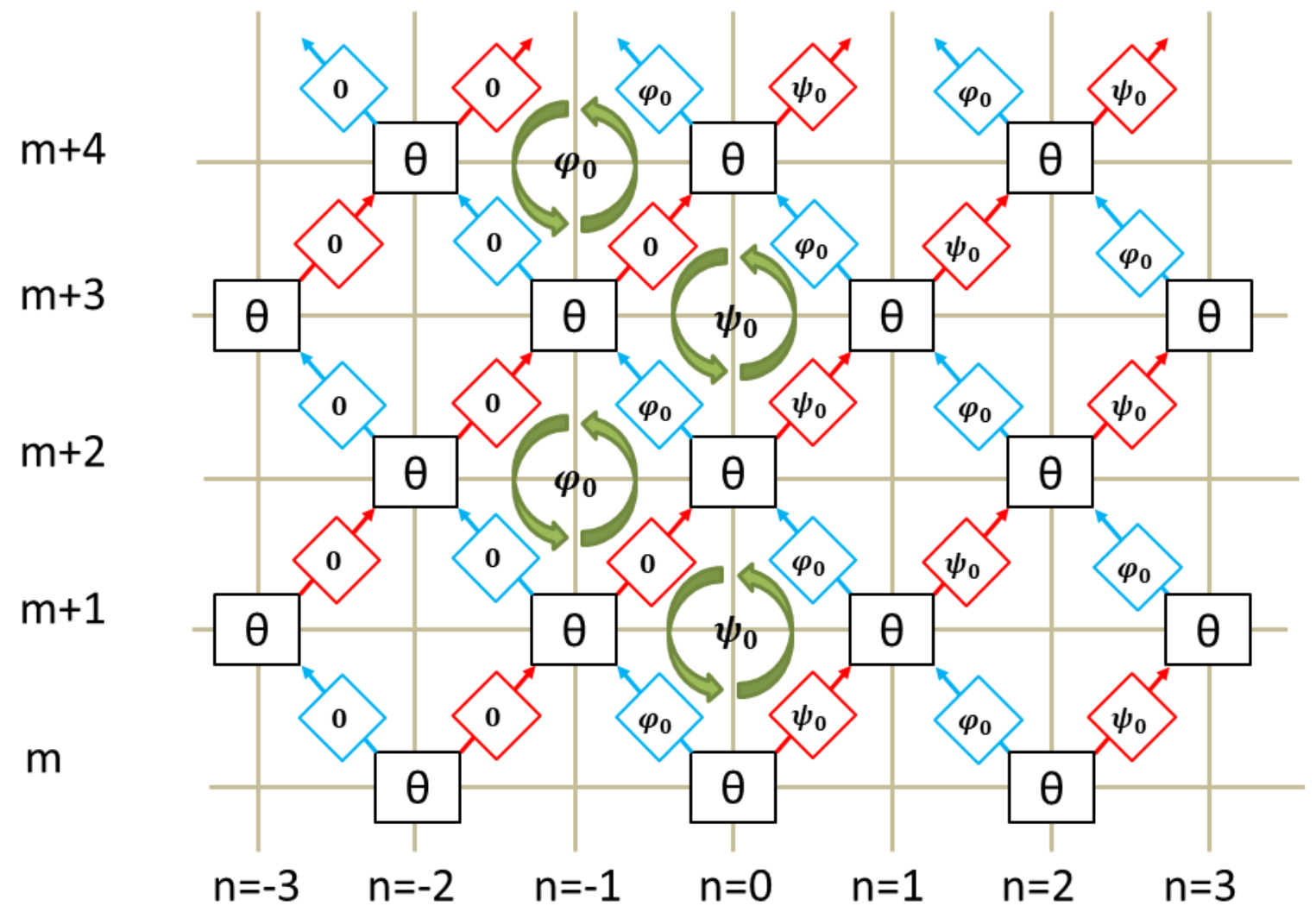

Figure 3. Scheme of phase accumulation (green circulating arrows) at the interface of gauge field jump.

\section{RESULTS AND DISCUSSION}

Calculating the mode structure of the system with an interface, a new type of localized modes reveals. While the bandgaps are identical on both sides of the interface, at the $\varphi=-\psi$ regime, interface modes appear inside the photonic gaps for any non-zero values of phase modulation (fig.4a). These modes turn out to be localized at the interface. 
Note that synchronous modulation, that is when $\varphi=\psi$, in some cases also leads to a presence of localized states, though such a modulation leads to vertical shift of propagation constant beta at right-hand side relative to left-hand side, thus decreasing the band-gap, and the area of localized modes existence (fig. 4b).
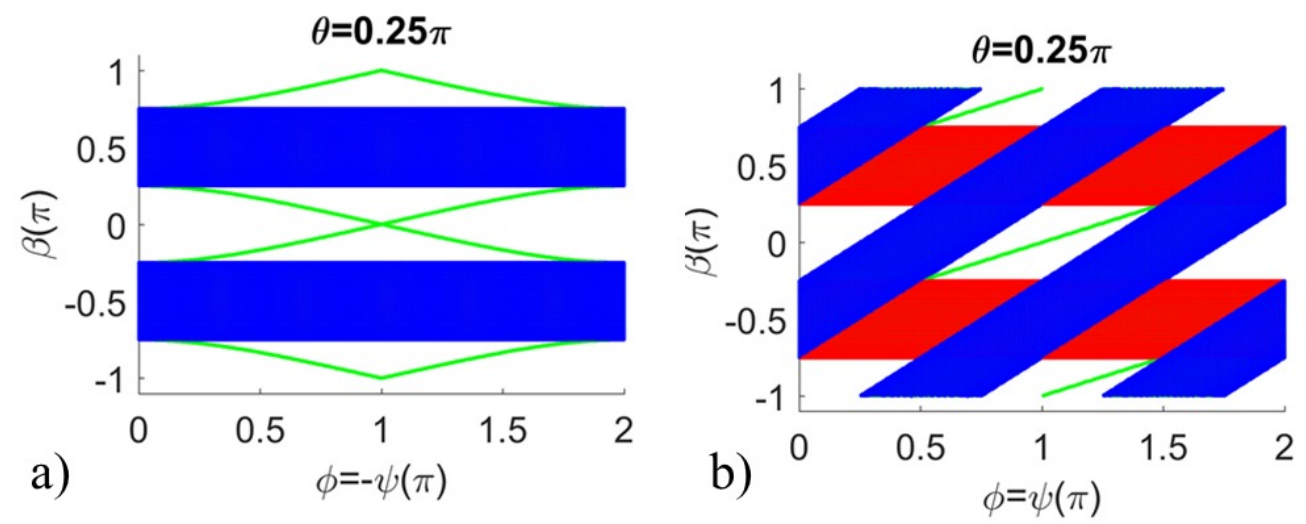

Figure 4. Propagation constant $(\beta)$ of areas from the left and from the right of the interface and of the modes with surface waves in $\varphi=-\psi$ regime (a) and in $\varphi=\psi$ regime (b). Red dots represent $\beta$ of left area, blue dots correspond to right area and green dots stand for $\beta$ of localized modes. In the regime (a) interface localized modes exist at any non-zero value of modulation.

We have calculated the localization strength on the left and right sides of the interface in the plane of phase modulation amplitudes $\left(\varphi_{0}, \psi_{0}\right)$ and observe that localization occurs in a broad parameter range (fig.5). This is in sharp contrast to Aharonov-Bohm photonic caging, which can happen only at particular resonant conditions [8]. Maximum localization corresponds to $\varphi_{0}=-\psi_{0}=\pi$.
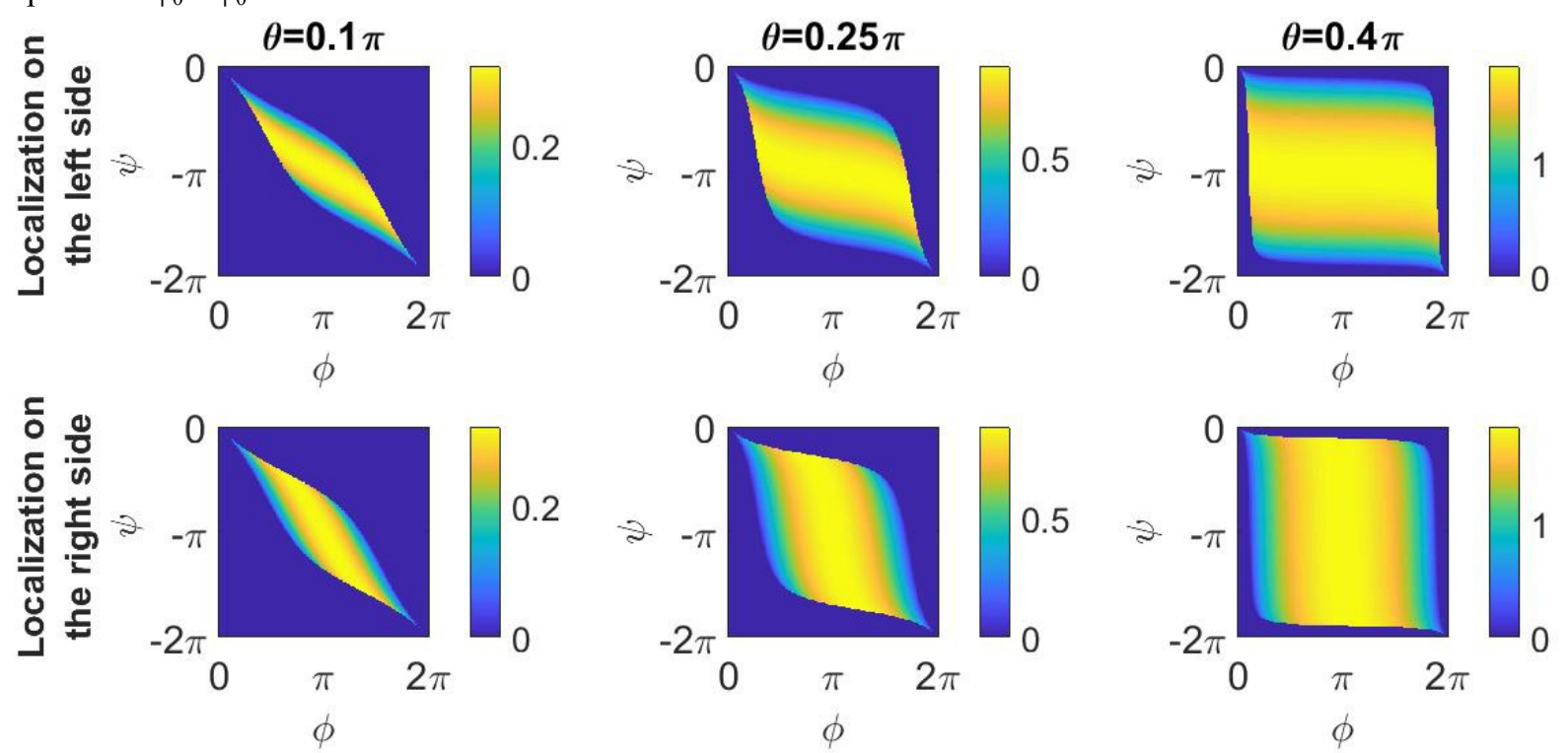

Figure 5. Localization strength of surface mode on the left and on the right sides of the interface at $\theta=0.1 \pi, 0.25 \pi, 0.4 \pi$.

We further establish that the interface modes are highly robust to variations of the lattice parameters, such as different amplitudes of phase modulators $\varphi_{0}$ and $\psi_{0}$, different coupler coefficient $\theta$ (fig.6). Increasing coupling coefficient $\theta$ results in shrinking transmission band, being equal to $\pi-2 \theta$, thus increasing the region of interface states. 

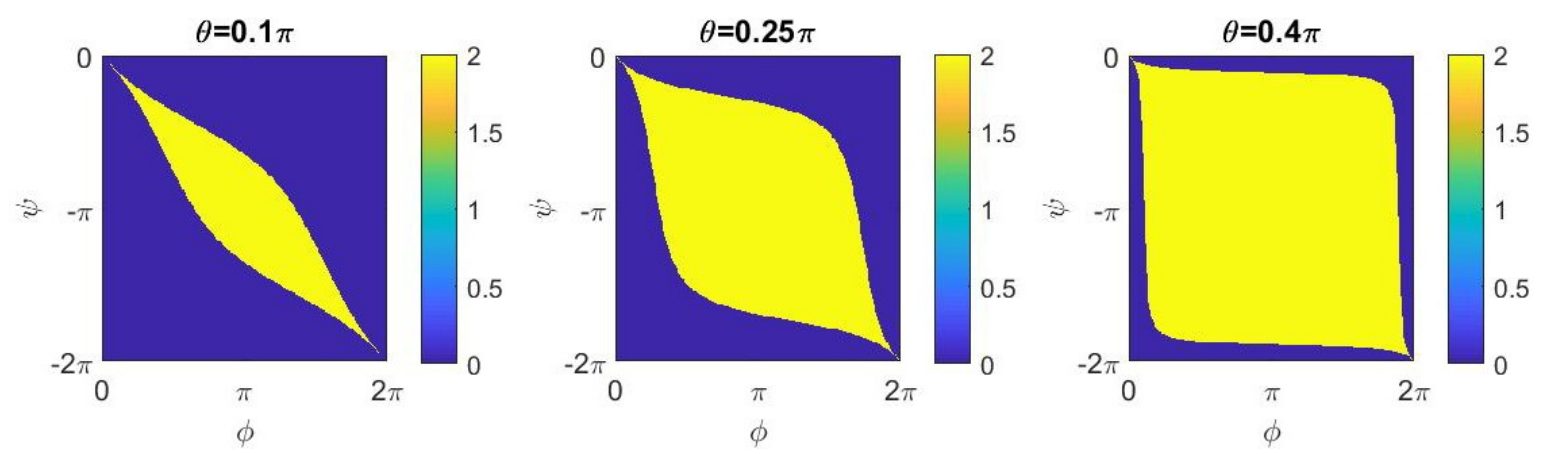

Figure 6. Number of modes localized at the interface depending on $\varphi_{0}, \psi_{0}$ and at different $\theta=0.1 \pi ; 0.25 \pi ; 0.4 \pi$.

\section{CONCLUSION}

We demonstrate a new type of localized mode supported by gauge field interface for $\varphi_{0}=-\psi_{0}$ in absence of band-gap shift. We show that this new localized modes are very robust to variations of lattice parameters and its maximum degree of localization corresponds to $\varphi_{0}=-\psi_{0}=\pi$ at any given coupling coefficient $\theta$. We anticipate that our predictions can initiate further theoretical and experimental studies of localized modes supported by effective magnetic fields at gauge interfaces in various photonic structures.

This work is supported by the Russian Science Foundation (16-12-10402). A.A.S. acknowledges support by the Australian Research Council (ARC) (DP160100619).

\section{REFERENCES}

[1] Lin, Q., Fan, S., "Light guiding by effective gauge field for photons," Phys. Rev. X, 4(3), 031031 (2014).

[2] Regensburger, A., Bersch, C., Miri, M., Onishchukov, G., Christodoulides, D. N., Peschel, U., "Parity-time synthetic photonic lattices," Nature 488(7410), 167-171 (2012).

[3] Schreiber, A., Cassemiro, K., N., Potoček, V., Gábris, A., Mosley, P., J., Andersson, E., Jex, I., Silberhorn, C., "Photons walking the line: a quantum walk with adjustable coin operations," Phys. Rev. Lett., 104(5), 050502 (2010).

[4] Regensburger, A., Bersch, C., Hinrichs, B., Onishchukov, G., Schreiber, A., Silberhorn, C., Peschel, U., "Photon propagation in a discrete fiber network: an interplay of coherence and losses," Phys. Rev. Lett., 107(23), 233902 (2011).

[5] Vatnik, I., D., Tikan, A., M., Churkin, D., V., Sukhorukov, A., A., "Anderson Localization In Optical Mesh Lattices Realized In Time Domain” The European Conference on Lasers and Electro-Optics, p. CD_P_8 (2015, June).

[6] Wimmer, M., Regensburger, A., Miri, M., A., Bersch, C., Christodoulides, D., N., Peschel, U., "Observation of optical solitons in PT-symmetric lattices," Nat. Commun., 6, 7782 (2015).

[7] Fang, K., Yu, Z., Fan, S., "Realizing effective magnetic field for photons by controlling the phase of dynamic modulation," Nat. Photon., 6(11), 782-787 (2012).

[8] Longhi, S., "Aharonov-Bohm photonic cages in waveguide and coupled resonator lattices by synthetic magnetic fields," Opt. Lett., 39(20), 5892-5895 (2014). 